\title{
A Combined Laboratory and Synchrotron In-Situ Photoemission Study of the Rutile $\mathrm{TiO}_{2}$ (110) / Water Interface
}

To cite this article before publication: Conor Byrne et al 2021 J. Phys. D: Appl. Phys. in press https://doi.org/10.1088/1361-6463/abddfb

\section{Manuscript version: Accepted Manuscript}

Accepted Manuscript is "the version of the article accepted for publication including all changes made as a result of the peer review process, and which may also include the addition to the article by IOP Publishing of a header, an article ID, a cover sheet and/or an 'Accepted Manuscript' watermark, but excluding any other editing, typesetting or other changes made by IOP Publishing and/or its licensors"

This Accepted Manuscript is @ 2021 The Author(s). Published by IOP Publishing Ltd.

As the Version of Record of this article is going to be / has been published on a gold open access basis under a CC BY 3.0 licence, this Accepted Manuscript is available for reuse under a CC BY 3.0 licence immediately.

Everyone is permitted to use all or part of the original content in this article, provided that they adhere to all the terms of the licence https://creativecommons.org/licences/by/3.0

Although reasonable endeavours have been taken to obtain all necessary permissions from third parties to include their copyrighted content within this article, their full citation and copyright line may not be present in this Accepted Manuscript version. Before using any content from this article, please refer to the Version of Record on IOPscience once published for full citation and copyright details, as permissions may be required. All third party content is fully copyright protected and is not published on a gold open access basis under a CC BY licence, unless that is specifically stated in the figure caption in the Version of Record.

View the article online for updates and enhancements. 


\title{
A Combined Laboratory \& Synchrotron In-Situ Photoemission Study of the Rutile $\mathrm{TiO}_{2}(110) /$ Water Interface
}

\author{
Conor Byrne ${ }^{1,2}$, Khadisha M. Zahra ${ }^{1,2}$, Simran Dhaliwal ${ }^{3,4}$, David C. Grinter ${ }^{4}$, Kanak \\ Roy $^{4}$, Wilson Q Garzon ${ }^{4}$, Georg Held ${ }^{4}$, Geoff Thornton ${ }^{3}$ and Alex S. Walton ${ }^{1,2}$ \\ ${ }^{1}$ Department of Chemistry, University of Manchester, M13 9PL, UK \\ ${ }^{2}$ Photon Science Institute, University of Manchester, M13 9PL, UK \\ ${ }^{3}$ London Centre for Nanotechnology and Chemistry Department, University College London, 20 \\ Gordon Street, London WC1H 0AJ, UK \\ ${ }^{4}$ Diamond Light Source, Harwell Campus, Didcot, UK \\ E-mail: alex.walton@manchester.ac.uk, conor.byrne@manchester.ac.uk
}

\begin{abstract}
In-situ analysis of the $\mathrm{TiO}_{2}$ / water interface via NAP-XPS is demonstrated in both a lab based system (NAP-cell configuration) and synchrotron endstation (backfill configuration). Ultra-thin wetting layers (UTWL) of liquid water $(\sim 10 \mathrm{~nm})$ are formed on a rutile $\mathrm{TiO}_{2}$ surface with minimal contamination present in addition to unique insight during the growth of the liquid films as indicated via NAP-XPS, in-situ sample temperature and background vapour pressure monitoring. Chemical changes at the solid / liquid interface are also demonstrated via healing of $\mathrm{Ti}^{3+}$ surface defect states. Photon depth profiling of the as grown liquid layers indicate that the formed films are ultra-thin $(-10 \mathrm{~nm})$ and likely to be continuous in nature. This work demonstrates a novel and flexible approach for studying the solid / liquid interface via NAP-XPS which is readily integrated with any form of NAP-XPS system, thereby making a critical interface of study available to a wide audience of researchers for use in operando electrochemical and photocatalytic research.
\end{abstract}

Keywords: NAP-XPS, AP-XPS, UTWL, TiO 2 , Rutile, solid/liquid interface, Offset Droplet, VerSoX

\section{Introduction}

Titanium dioxide has long been recognised as an important photocatalytic material with diverse applications in pollution remediation, self-cleaning surfaces and solar water splitting ${ }^{1-3}$. A detailed understanding of $\mathrm{TiO}_{2}$ surface chemistry is critical to development in these areas, and such the chemistry of $\mathrm{TiO}_{2}$ surfaces has been intensively studied.

The vast majority of these studies of the surface reactivity and structure of various $\mathrm{TiO}_{2}$ phases have taken place in ultra-high vacuum (UHV) environments, in order to both maintain a controllable environment for surface reactions and due to pressure limitations in relation to the surface analysis techniques used ${ }^{4,5}$. Problematically, $\mathrm{TiO}_{2}$ photocatalysis occurs at the solid/liquid interface making the connection of experimental results obtained within UHV conditions to the real-world catalysis challenging ${ }^{6,7}$.

X-ray photoelectron spectroscopy (XPS) has been routinely used in the study of $\mathrm{TiO}_{2}$ surfaces, owing to the inherent elemental and rich chemical information obtainable from the surface region of the sample (typically 5 to 10 $\mathrm{nm})^{8,9}$. Typically, XPS analysis has been restricted to UHV conditions due to limitations of both the X-ray source and electron energy analyser requiring at least high vacuum conditions during operation in addition to prevention of scattering of the emitted photoelectrons prior to analysis. Near ambient pressure (NAP) XPS has been pursued in order to overcome this pressure gap limit, offering the ability to 
study surfaces and interfaces with background gas up to pressures of $\sim 20$ mbar (depending on various experimental conditions $)^{10}$. This mbar range of afforded background pressure coincides with the vapour pressure of water ( 23 mbar at room temperature) making the study of liquid water using NAP-XPS feasible ${ }^{11}$. Further still, the ability to form ultra-thin wetting layers (UTWL) of liquid water in the 10 $\mathrm{nm}$ thickness range has been previously demonstrated, thereby enabling the study of the solid / liquid interface via NAP-XPS ${ }^{12,13}$.

Although the study of the solid / liquid interface via NAPXPS has previously been demonstrated, typically this requires complex vacuum manipulators, synchrotron light sources and is limited to restrictively long samples $(\sim 2 \times 5$ $\mathrm{cm})^{14-16}$. These limitations preclude work on single crystals and also make preparing well characterised surfaces via sputter/annealing or other UHV-based surface science techniques difficult or impossible. The development of a method to study the solid / liquid interface which requires minimal set-up and is portable between laboratory-based and synchrotron NAP-XPS systems with the ability to study standard sample geometries would be of enormous benefit to researchers globally. In this study, we present a method of probing the solid / liquid interface between a rutile $\mathrm{TiO}_{2}$ (110) surface and varying thicknesses of liquid water with surface and chemically sensitive NAP-XPS. This method, previously described as the "Offset Droplet" method ${ }^{13}$, is shown to be compatible with both laboratory and synchrotron NAP-XPS systems, which employ a NAP-Cell and a backfill-type arrangement, respectively. Using this method, we report NAP-XPS results for the rutile (110) $\mathrm{TiO}_{2}$ / liquid water interface. This development is a critical step towards operando studies of $\mathrm{TiO}_{2}$-based photocatalysts

\section{Experimental Details}

A single crystal rutile $\mathrm{TiO}_{2}$ (110) sample was used throughout the course of this study. The surface of the single crystal was prepared in ultra-high vacuum (UHV) conditions by repeated cycles of Ar ion sputter cleaning $(1 \mathrm{kV}, 30 \mathrm{~mA})$ and annealing $\left(700^{\circ} \mathrm{C}\right)$.

Milli-Q water was used as a dispensing liquid throughout this study (resistivity of $18.2 \mathrm{M} \Omega$ and $<10 \mathrm{ppb}$ hydrocarbon contamination). The liquid water was outgassed in a dedicated custom vacuum rig (see SI for further details) prior to insertion and subsequent analysis in the NAP-XPS system(s). This outgassing procedure produces ultra-stable liquid droplets during analysis within the vacuum environment of the NAP-XPS system(s) used throughout the course of this study and ensured minimal contamination present in the liquid.

Two NAP-XPS analysis systems were used within this study. The first, based at the University of Manchester
(UoM), is a SPECS DeviSim NAP-XPS system which utilises a UHV environment (base pressure $5 \times 10^{-10}$ mbar) for sample preparation and analysis in addition to a NAP cell which docks onto the first differential pumping aperture of a PHOIBOS 150 NAP differentially pumped electron energy analyser. The NAP cell allows near ambient pressure analysis of a sample while maintaining a UHV environment in the main analysis chamber which also houses a monochromatic Al $\mathrm{K \alpha}$ source $(1487 \mathrm{eV})$. The monochromatic $\mathrm{Al} \mathrm{K} \alpha$ source was micro-focussed to an analysis spot size of $300 \mu \mathrm{m}$ on the sample surface and used to excite the photoelectrons during experimentation. Analysis of the photoelectrons was undertaken by use of the PHOIBOS 150 NAP analyser operating at a pass energy of $30 \mathrm{eV}$ and utilising a $300 \mu \mathrm{m}$ diameter aperture cone. The sample surface plane was positioned at $90^{\circ}$ to ground level during analysis as dictated by the system geometry. The nominal energy resolution of the system was $0.5 \mathrm{eV}$, as measured via the $A g 3 \mathrm{~d}_{5 / 2}$ peak of a sputter cleaned silver sample.

The second NAP-XPS system used was the AmbientPressure endstation of the VerSoX (B07) beamline located at the Diamond Light Source in Oxfordshire, $\mathrm{UK}^{17}$. The endstation utilises synchrotron radiation, generated from a bending magnet and a collimated plane grating monochromator to deliver a variable photon energy of between 250-2800 eV. Three photon energies were used in this study namely; $1487 \mathrm{eV}, 2000 \mathrm{eV}$ and $2400 \mathrm{eV}$. The NAP analysis chamber is of the "backfill" configuration and utilised a vacuum transfer system to port the sample between a dedicated UHV sample preparation chamber and the analysis chamber. The sample preparation chamber contains sputter/anneal facilities as well as a low energy electron diffraction (LEED) instrument. Photoelectrons were analysed using an identical SPECS PHOIBOS 150 NAP differentially pumped analyser as that in the University of Manchester system (with a similar $300 \mu \mathrm{m}$ diameter aperture analysis cone). The sample surface plane was positioned at $30^{\circ}$ to ground level during analysis as dictated by the system geometry. All spectra obtained from the B07 beamline were binding energy calibrated to the $\mathrm{Ti} 2 \mathrm{p}_{3 / 2}$ line at $459.5 \mathrm{eV}$, calculated from the analysis of the "As Loaded" condition if the $\mathrm{TiO}_{2}$ crystal from the University of Manchester fixed energy (Al $\mathrm{K} \alpha 1487 \mathrm{eV}$ ) source system. The nominal resolution of the system is noted to be $0.5 \mathrm{eV}^{17}$.

Liquid water was introduced into both NAP-XPS systems via the use of ThermoFisher Scientific "nanoViper" capillaries, utilising custom vacuum feedthroughs fabricated using viper-union components which were welded to a CF16 flange to create a UHV compatible liquid feedthrough. These liquid inlet lines are capable of 400 bar internal liquid pressures and utilise an inner diameter of $90 \mu \mathrm{m}$. The capillaries consist of an inner silica core with an outer PEEK 
covering and have been found to be UHV compatible as well as bake-able within UHV systems (to a maximum temperature of $120{ }^{\circ} \mathrm{C}$ ). The liquid dispensing capillary end (visible in Figure 1 below) was fabricated by cutting a complete nanoViper capillary to the desired length to position the end such that it was located immediately above the sample surface and adjacent to the analysis cone (offset by $\sim 2 \mathrm{~mm}$ ) during sample alignment and analysis.

Precision control of the liquid flow rate and pressure within the nanoViper lines was achieved through the use of a dual piston Vindum Engineering metering pump. This pump allows precision control of liquid flow rates down to 0.1 $\mu \mathrm{l} / \mathrm{min}$ and is capable of pressures of up to $400 \mathrm{bar}$. The liquid containing pistons of the pump are vacuum compatible (leak tested to $1 \times 10^{-6} \mathrm{mbar}$ ), allowing storage of outgassed liquids for up to one week without the need to replenish or further outgas the liquid.

No gross issues were noted in relation to the droplet controllability and sample angle for each system. Owing to the small droplet size used ( $1 \mathrm{~mm}$ diameter), located on a flat and UHV cleaned surface, we expect (and observe) that gravitational effects to have a minimal impact on droplet stability when compared to wetting and capillary forces at the dimensions used in this study.

Throughout the course of this study, the sample was actively cooled to $\sim 6^{\circ} \mathrm{C}$ prior to liquid insertion in order to reduce the background vapour pressure during NAP-XPS analysis. Cooling was performed by using an external chiller to actively cool the atmosphere side of the sample stage on both systems during analysis.

XPS peak fitting analysis was carried out using both Aanalyzer and CasaXPS peak fitting software packages. Line shapes were fitted utilising Voigt profiles with Shirley backgrounds. All spectra were fitted concurrently in order to ensure consistency of peak fitting parameters across the sample data sets.

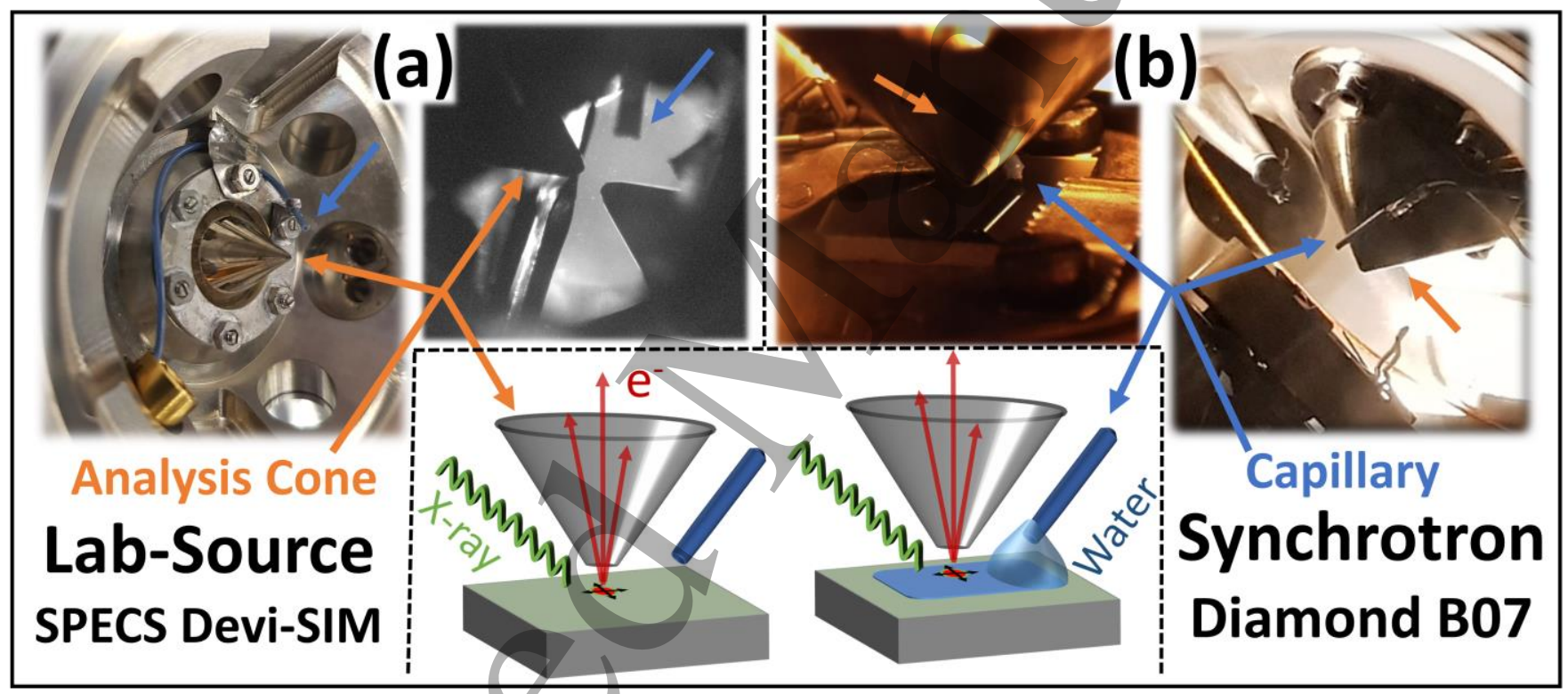

Figure 1: Overview of the Offset droplet technique for (a) capillary set-up within a lab based NAP-cell configuration and (b) capillary set-up within the NAP backfill analysis chamber of the Diamond synchrotron VerSoX beamline. NAP-XPS analysis cone for each system is indicated with an orange arrow and liquid capillary lines are indicated with blue arrows.

\section{Results \& Discussion}

\section{University of Manchester SPECS DeviSim System:}

The $\mathrm{TiO}_{2}$ rutile (110) single crystal sample was loaded into the UHV portion of the University of Manchester lab based NAP-XPS system and sputter annealed using standard UHV preparation methods as described in previous reports ${ }^{6}$. Following sample preparation, the NAP cell was docked onto the first differential aperture of the analyser and the sample subsequently transferred into the cell and sealed. All cleaning and sample transfer were conducted under UHV conditions. Survey, Ti 2p, O 1s and C 1s spectra for all experimental stages are depicted in Figure 2-5, respectively.

Post UHV sputter annealing (As Loaded), the Ti 2p spectrum (Figure 3 (a)) shows a strong doublet (with the Ti $2 \mathrm{p}_{3 / 2}$ component centred at $459.5 \mathrm{eV}$ ) consistent with the $\mathrm{TiO}_{2}$ substrate signal expected from the rutile surface ${ }^{8}$. In addition, a small lower binding energy shoulder was evident in the $\mathrm{Ti} 2 \mathrm{p}_{3 / 2}$ spectrum $(457.6 \mathrm{eV})$, consistent with a low concentration of $\mathrm{Ti}^{3+}$ surface sub-oxide states as seen in

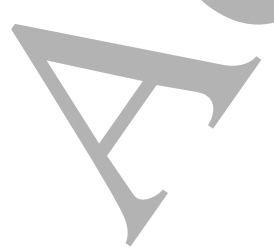


previous studies 9 . The $\mathrm{O}$ 1s spectrum for the "As Loaded" $\mathrm{TiO}_{2}$ surface (Figure 4 (a)) indicates a strong lattice oxide peak for $\mathrm{TiO}_{2}(530.7 \mathrm{eV})$ in addition to a small higher binding energy shoulder $(531.8 \mathrm{eV})$ attributed to $\mathrm{OH}$ surface groups $^{18}$. The C 1 s spectrum (Figure 5 (a)) also indicates a minor amount of adventitious carbon present on the surface of the sample.

Following "As Loaded" analysis, Milli-Q water was then dispensed onto the surface of the $\mathrm{TiO}_{2}$ crystal through the insitu capillary set-up (flow rate of $5 \mu \mathrm{l} / \mathrm{min}$ ), with the liquid droplet localised adjacent to the analysis area in order to create a background water vapour pressure above the analysis region. As can be seen in the Ti $2 p$ spectrum for the sample within a background of water vapour (Figure 3 (b)), the sub-oxide $\mathrm{Ti}^{3+}$ state (as seen in Figure 3 (a)) is not observed, consistent with the relatively high localised water vapour pressure (6 mbar, as measured using in-situ cell pressure gauge) healing the surface defect states (overlaid $\mathrm{Ti}$ $2 \mathrm{p}$ spectra available in the $\mathrm{SI}$ ), as demonstrated in previous ex-situ literature studies ${ }^{19}$. The $\mathrm{O} 1 \mathrm{~s}$ spectrum (Figure 4 (b)) also exhibits changes during water vapour exposure, as seen with the appearance of a peak at $535.7 \mathrm{eV}$ which is attributed to $\mathrm{H}_{2} \mathrm{O}$ in the vapour phase between the sample and analysis cone ${ }^{12,13}$.

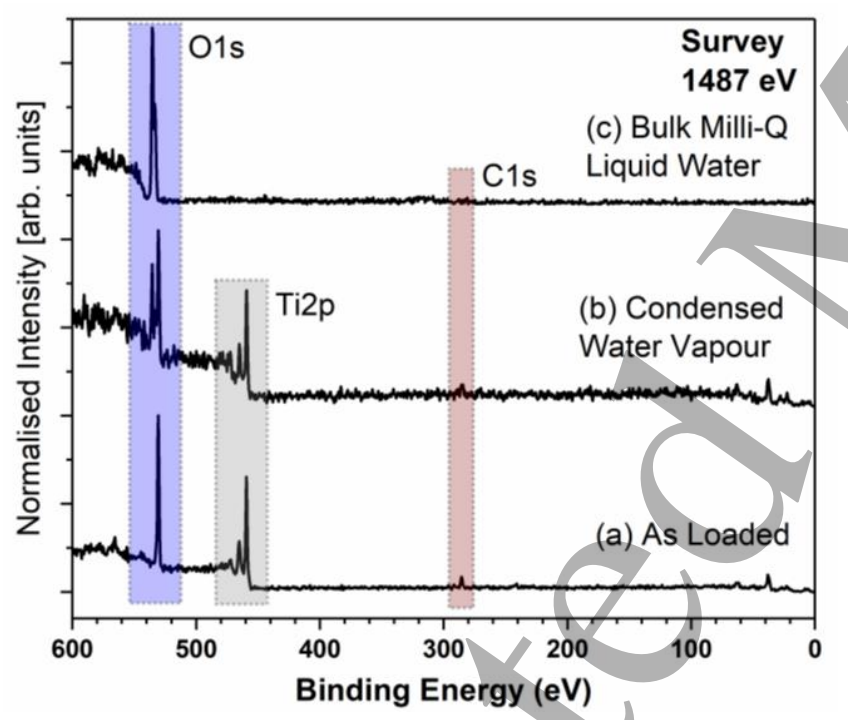

Figure 2: UoM NAP-XPS Al K $\alpha$ ( $h v=1487 \mathrm{eV})$ survey spectra for $\mathrm{TiO}_{2}$ (110) (a) As loaded in NAP-Cell, (b) Condensed Water Vapour and (c) Bulk Milli-Q Liquid Water. Survey spectra are each normalised from min to max.

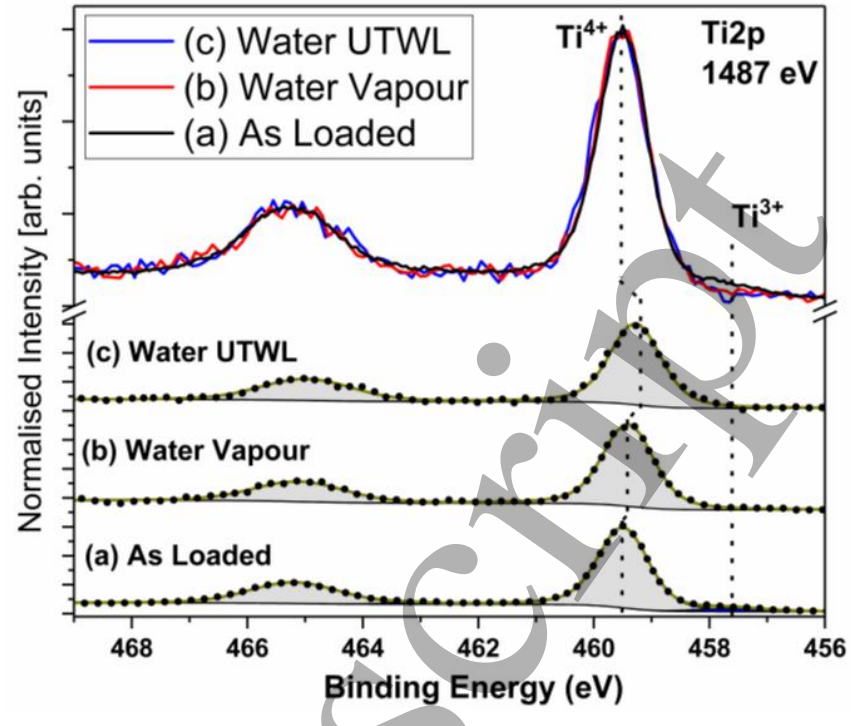

Figure 3: UoM NAP-XPS AI K $\alpha$ ( $h v=1487 \mathrm{eV}$ ) Ti $2 \mathrm{p}$ spectra of (a) As Loaded, (b) Water Vapour and (c) Water UTWL experimental stages. All spectra are normalised to the Ti $2 p_{3 / 2}$ signal. Overlay spectra are charge corrected to the "As Loaded" Ti $2 p_{3 / 2}$ signal, highlighting the relative change in the $\mathrm{Ti}^{3+}$ chemical state.

A separate small shoulder peak at higher binding energy relative to the lattice peak is also observed at $533.2 \mathrm{eV}$ which is consistent with condensed water vapour on the surface of the $\mathrm{TiO}_{2}$ erystal ${ }^{13,18}$. A decrease in the $\mathrm{C} 1 \mathrm{~s}$ signal (in addition to the raw $\mathrm{Ti} 2 \mathrm{p}$ and $\mathrm{O} 1 \mathrm{~s}$ signals) is also noted as evident in Figure 5 (b), consistent with the increase in background water vapour pressure which acts to reduce the overall signal obtained for the sample surface, typical of all NAP-XPS experiments ${ }^{17}$. The liquid droplet was then advanced over the analysis region by increasing the flow rate through the capillary (flow rate of $15 \mu \mathrm{l} / \mathrm{min}$ ) in order to form a layer of bulk liquid water under the analysis cone which was subsequently analysed via NAP-XPS. As can be seen in the survey scan of the bulk Milli-Q water (Figure 2 (c)), the only evident element is oxygen (as hydrogen in not detectable via XPS analysis) which when seen in the highresolution $\mathrm{O} 1 \mathrm{~s}$ window (Figure 4 (d)) consists of two separate peaks attributed to water vapour and liquid water. A minor C 1s signal was however noted upon prolonged scanning of the narrow window $\mathrm{C}$ 1s region (Figure 5 (d)). This small signal is testament to the cleanliness of the water source used and also to the upgraded materials (as detailed in the Experimental Details section) used in the capillary set-up compared to first reports of the Offset Droplet technique ${ }^{13}$. These upgraded materials consist of a capillary with internal silica coating (external PEEK coating) in place of the stainless steel capillary previously used, a new liquid outgassing vacuum setup (see SI) compared to the previously used He sparging method and a new liquid pump (Vindum 
Engineering metering pump) which is vacuum compatible, capable of storing and maintaining liquids in a clean and outgassed state, upgraded from an Agilent 1260 Infinitiy HPLC pump.

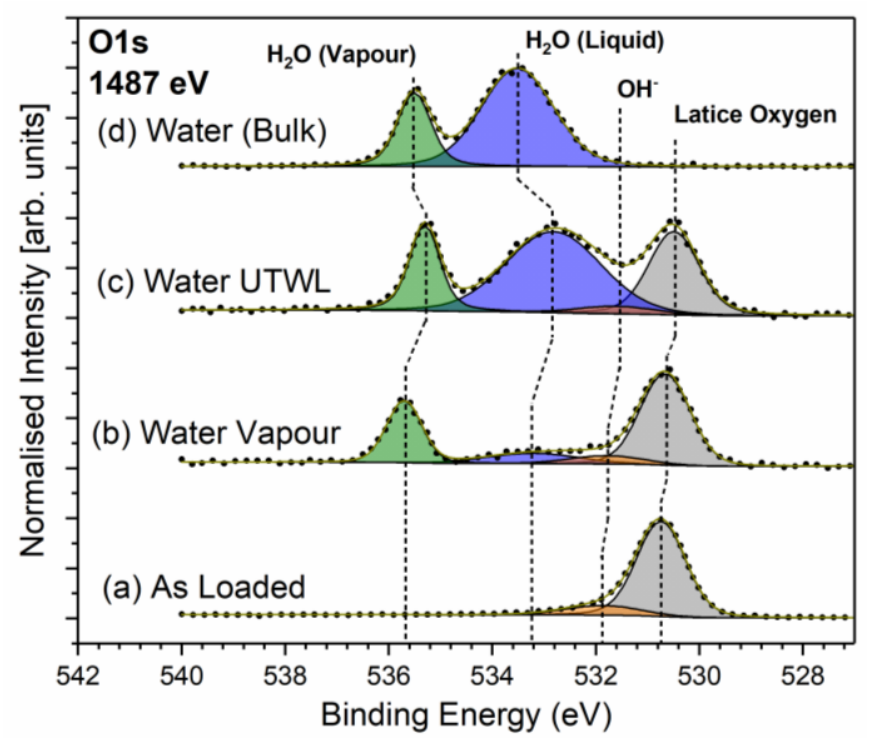

Figure 4: UoM NAP-XPS Al K $\alpha$ ( $h v=1487 \mathrm{eV}) \mathrm{O}$ 1s spectra of (a) As Loaded, (b) Water Vapour, (c) Water UTWL and (d) Water (Bulk) experimental stages. All spectra are normalised to the lattice oxygen signal with the exception of the "Water (Bulk)" scan which is normalised to the liquid water signal of the "Water UTWL" scan.

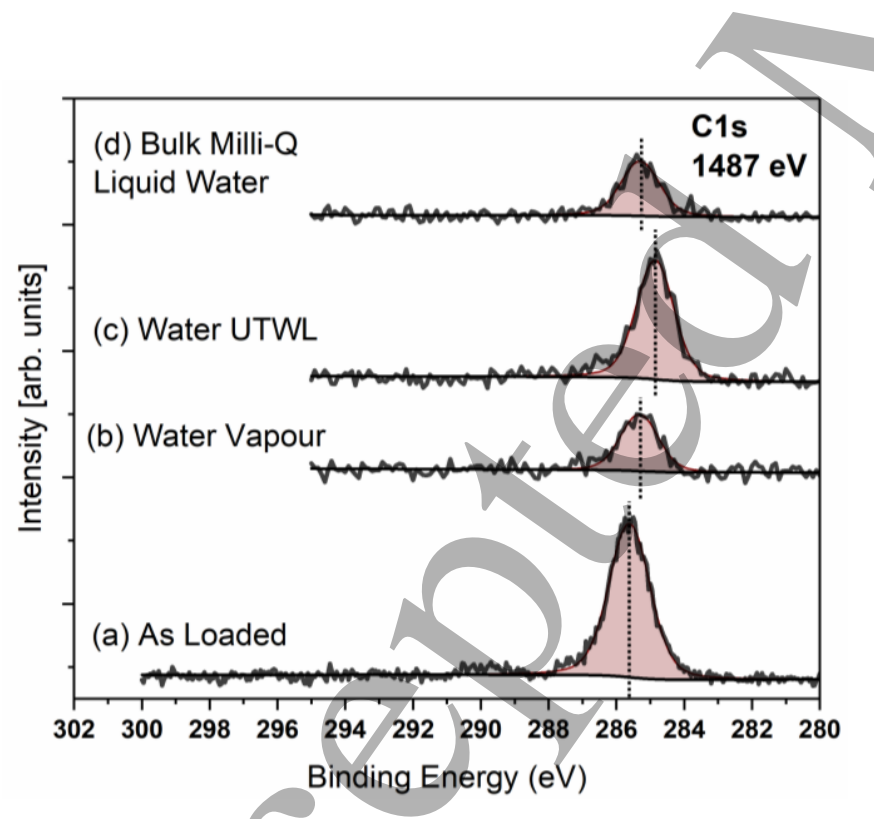

Figure 5: UoM NAP-XPS Al K $\alpha(\mathrm{hv}=1487 \mathrm{eV})$ raw data for the $C$ 1s XPS region showing the level of carbon contamination throughout the course of the experiment.
The bulk water droplet was then receded from the analysis area in order to form an ultra-thin wetting layer of liquid water on the $\mathrm{TiO}_{2}$ surface to examine the solid / liquid interface. Associated core level spectra for this experimental stage can be seen in the Ti 2 p, O $1 \mathrm{~s}$ and $\mathrm{C} 1 \mathrm{~s}$ high resolution spectra (Figure 3-5 (c), respectively). Most obviously, a change from the initial vapour condensate stage is seen in the O 1s spectra of Figure 4 (c) with a growth of the liquid water peak relative to the lattice oxygen signal (indicating a thicker layer of liquid water on the sample surface). Substrate suppression calculations (as outlined in the SI) which were performed on the raw $\mathrm{Ti} 2 \mathrm{p}$ spectra for both vapour condensate and UTWL experimental stages indicate a relative growth of $\sim 2.2 \mathrm{~nm}$ of liquid water on the surface of the sample following advancing and receding of the liquid droplet. No change to the ratio of $\mathrm{OH}$ surface groups to $\mathrm{TiO}_{2}$ lattice peak was noted throughout the course of the experiment. Although previous demonstrations have shown it is possible to obtain a larger thickness of water using this method (up to $17 \mathrm{~nm})^{13}$, here we demonstrate the ability to also form relatively thinner layers of liquid water. The capability to control UTWL thickness means that our methodology allows us to obtain the best compromise between/good signal from the solid/liquid interface and a reasonable water film thickness for representative interfacial chemistry. A small growth in the C 1s spectra (Figure 5 (c)) is also noted indicating only a minor increase in carbon following the formation of the ultra-thin wetting layer. It should also be noted that within the $\mathrm{C} 1 \mathrm{~s}$ spectra, the majority of the signal appears to originate from $\mathrm{C}-\mathrm{C}$ bonds with only very minor (if any) $\mathrm{C}-\mathrm{O}$ or $\mathrm{C}=\mathrm{O}$ bonds. These bonding states within the $\mathrm{O} 1 \mathrm{~s}$ spectra typically overlap with the $\mathrm{OH}$ signal, however peak fits indicate no increase in $\mathrm{OH}$ (or $\mathrm{C}=\mathrm{O}, \mathrm{C}-\mathrm{O}$ groups) over the course of the experiment, again indicating high control of the levels of contamination during exposure of the sample surface to liquid water.

Further to the chemical and compositional changes to the interface (both healing of the $\mathrm{Ti}^{3+}$ surface states and growth of liquid water on the surface), an apparent binding energy shift is noted in the spectra throughout the course of the experiment. As shown in both $\mathrm{Ti} 2 \mathrm{p}$ and $\mathrm{O} 1 \mathrm{~s}$ spectra, the $\mathrm{TiO}_{2}$ substrate peaks shift to lower binding energy as a result of both the condensed water vapour and the UTWL formed on the surface. A similar result was seen previously on a $\mathrm{TiO}_{2}$ material using the Dip and Pull technique and explained via band bending at the surface of the $\mathrm{TiO}_{2}$ when in contact with a liquid over layer ${ }^{20}$, supported also by similar solid / liquid interface analysis results in the literature ${ }^{21}$. In addition, similar shifts are also noted in the $\mathrm{C} 1 \mathrm{~s}$ and for both vapour and liquid water $\mathrm{O}$ 1s peaks for the UTWL. The liquid and vapour $\mathrm{H}_{2} \mathrm{O}$ peaks also appear at lower binding energy with respect to the bulk liquid peaks, further indicating the 
interaction between the UTWL water film and underlying $\mathrm{TiO}_{2}$ surface.

\section{Diamond $\mathrm{B} 07$ results of $\mathrm{TiO}_{2}$ with water droplet:}

In order to investigate the $\mathrm{TiO}_{2} /$ water interface in more detail and to test the compatibility of the Offset Droplet technique at a synchrotron source, the VerSoX (B07) beamline at Diamond Light Source was utilised to host the capillary set-up in order to study the same $\mathrm{TiO}_{2} /$ water interface as that shown previously within the University of Manchester system.

Figure 6 shows camera images of the NAP-XPS analysis chamber with highlighted NAP-XPS analysis cone, $\mathrm{TiO}_{2}$ single crystal sample and nanoViper capillary during experimentation. The capillary can be seen to the bottomright of the cone both (a) before (at vacuum conditions) and (b) during solid/liquid interface analysis with liquid water droplet adjacent to the analysis area. Sample thermocouple connections can also be seen in the background of the image. They were used to track the sample temperature throughout the course of the experiment.
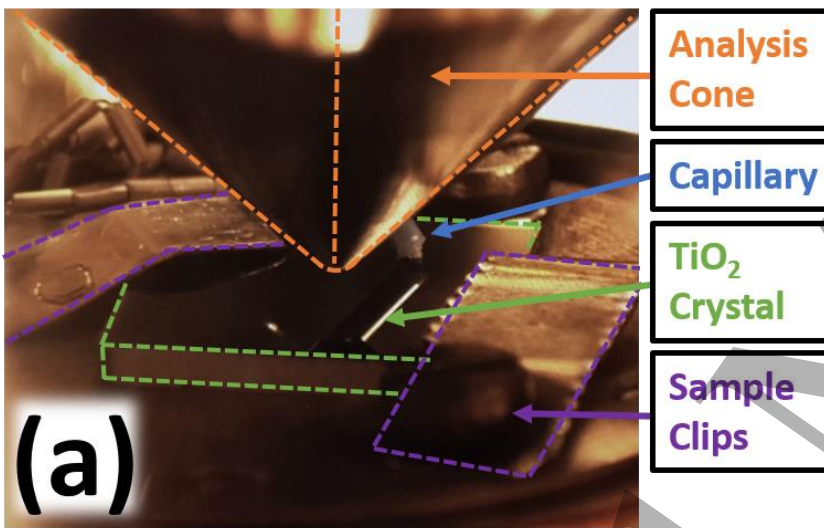
Cone

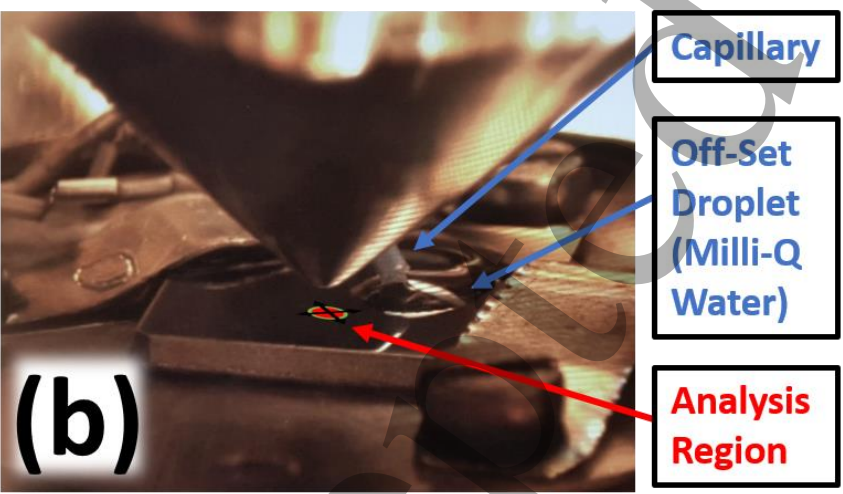

Figure 6: Camera images of the VerSoX endstation analysis chamber for: (a) As Loaded $\mathrm{TiO}_{2}$ sample analysis under vacuum conditions; and (b) Offset Droplet during the UTWL experiments. The sample surface and camera angle relative to ground level is $30^{\circ}$, allowing a horizontal view onto the plane of the sample surface.
As described previously, the rutile $\mathrm{TiO}_{2}$ (110) single crystal was cleaned utilising the dedicated UHV preparation chamber at the VerSoX endstation of the B07 beamline prior to vacuum transfer and insertion into the analysis chamber for UTWL experiments. Upon loading into the analysis chamber, the sample was analysed via XPS survey, Ti $2 p, O$ $1 \mathrm{~s}$ and $\mathrm{C} 1 \mathrm{~s}$ spectra. Liquid water was then dispensed onto the surface of the sample (flow rate $5 \mu \mathrm{l} / \mathrm{min}$ ), contained adjacent to the analysis region, in order to obtain spectra of the sample in background water vapour. In contrast to the "advance and recede" technique employed at the University of Manchester system (as outlined previously), the droplet was instead restrained to be adjacent to the analysis region in order to track the change in thickness of liquid water within the analysis region as a function of time. This was performed to assess the ability of the Offset Droplet technique to form ultra-pure water layers over the analysis region, with even lower levels of contamination than obtained previously.

Figure 7 displays both survey and Ti $2 p$ (insert) spectra of the $\mathrm{TiO}_{2}$ surface over the course of the experiment. The "As Loaded" survey scan indicates only very low quantities of residual surface carbon in addition to Argon (Ar $2 \mathrm{~s} \sim 320 \mathrm{eV}$ and $\operatorname{Ar} 2 \mathrm{p} \sim 243 \mathrm{eV}^{22}$ ), typical as a result of the sputter cleaning process. As can be seen, a decrease in counts overall is noted from the stages of the "As Loaded" condition (at vacuum), water vapour background (droplet adjacent to analysis area) and finally UTWL formation over the surface of the sample (droplet remaining adjacent to analysis area over a prolonged time). The relative decrease of the $\mathrm{Ti} 2 \mathrm{p}$ signal between the initial water vapour background and UTWL stages (as calculated in the SI) indicate the relative growth of a liquid water layer on the surface with a thickness of $12.4 \mathrm{~nm}$. 
2



Figure 7:VerSoX NAP-XPS (hv=1487 eV) raw intensity survey and $\mathrm{Ti} 2 \mathrm{p}$ (insert) spectra of the $\mathrm{TiO}_{2}(110)$ sample during in-situ liquid water exposure.

Figure 8 displays a colour intensity map of the $\mathrm{O} 1 \mathrm{~s}$ signal (normalised to the $\mathrm{TiO}_{2}$ lattice peak) during the time when the liquid water droplet was contained adjacent to the analysis area. The plot contains 80 scans which were spread evenly over the course of approximately 90 minutes in duration. It can also be noted that liquid water is present on the surface of the sample even at Scan 1, already occurring from the adjacent liquid droplet. As can be seen, a sharp increase in the liquid water thickness was noted at Scan 44 to Scan 50 , relative to the already present liquid signal of Scan 1. This sharp increase in liquid water thickness was also accompanied by a sharp increase in the sample temperature from $\sim 8^{\circ} \mathrm{C}$ to $10.4{ }^{\circ} \mathrm{C}$. It was also noted that the pressure within the chamber rose from the starting condition of 7.5 mbar at Scan 1 to 9.3 mbar at Scan 80 . Thus it can be seen that the growth of the liquid water layer, resulting in the 12.4 $\mathrm{nm}$ thickness, appears to have occurred in at least two stages. The initial growth stage, when the droplet was dispensed adjacent to the analysis area and subsequently scanned in Scan 1 and the second growth at Scan 44 where a sharp increase in liquid thickness was noted, with corresponding increases in temperature and background vapour pressure.

Two possible mechanisms can be proposed for the sharp growth of the liquid water on the surface of the $\mathrm{TiO}_{2}$ crystal. The first mechanism describes the growth as the spreading of the tail end of the droplet into the analysis region of the sample from the adjacent liquid droplet. This spreading of the liquid film would account for the increase in liquid thickness within the analysis region and is consistent with the growth mechanism described in the literature in relation to the "Dip \& Pull" technique ${ }^{12}$.



The second possible mechanism is a condensation mechanism. With the liquid droplet contained adjacent to the analysis region, the background water vapour could condense onto the surface of the sample, thereby creating a thicker film of liquid water. The noted increase in background vapour pressure (from 7.5 to 9.3 mbar) could induce a condensation of liquid water on the sample surface. In addition, the noted sharp temperature increase also favours this mechanism, described as heating of the sample surface via condensation (as opposed to cooling of the surface via evaporative cooling of water from the adjacent droplet). Previous NAP-XPS reports of forming thin layers of liquid water on surfaces have also shown a condensation mechanism is possible ${ }^{23}$.

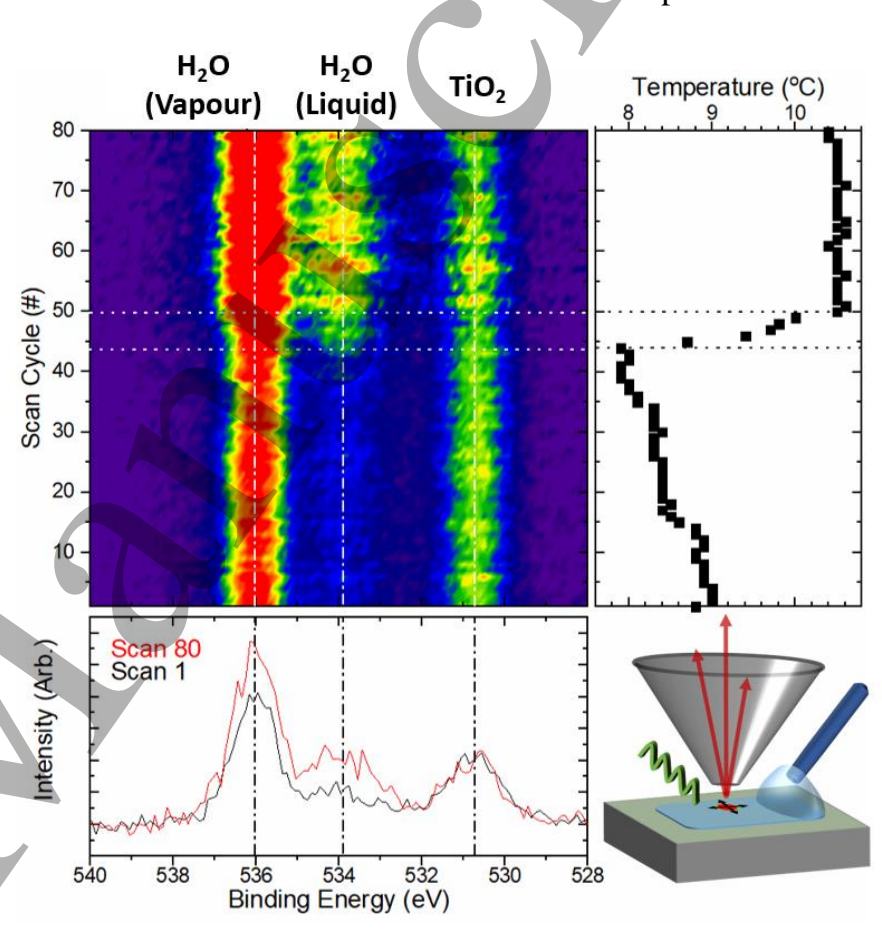

Figure 8: Colour map tracking the change in the XPS ( $h v=1487 \mathrm{eV}$ ) $\mathrm{O}$ 1s spectra (normalised to the $\mathrm{TiO}_{2}$ lattice peak) during prolonged exposure to the adjacent liquid water droplet at the VerSoX NAP-XPS system. The sample temperature profile for each scan is also plotted showing a sharp increase in temperature coinciding with the sharp increase in water thickness.

Figure 9 (a) displays the O 1s spectra of the "As Loaded" $\mathrm{TiO}_{2}$ (110) (1487 eV photon energy, at high-vacuum conditions) in addition to high quality narrow window scans of the final UTWL formed at three different photon energies (Figure 9 (b-d)). Increasing the photon energy while observing the emitted photoelectron signals allows conclusions about the UTWL to be drawn. The data plotted have been normalised to the $\mathrm{TiO}_{2}$ substrate lattice peak for all spectra. This makes it possible to track any changes to the liquid water overlayer signal as a result of photon energy depth profiling. The liquid water signal decreases relative to 
the lattice peak upon increasing photon energy (overlaid spectra available in the SI), indicating that the UTWL formed is consistent with a continuous liquid layer across the analysis region as opposed to isolated thick patches of liquid on the $\mathrm{TiO}_{2}$ surface. If thick isolated islands of liquid water (thicker than the information depth of XPS) instead were present on the sample surface, no change in the ratio of liquid water to $\mathrm{TiO}_{2}$ lattice peak intensity would be expected. Although not definitive proof of a continuous thin film, as we cannot rule out isolated thin film liquid islands or Stransky-Krastanov like growth, the photon depth profiling can rule out a regime where there is only thick (greater than information depth of XPS) islands of liquid water on the $\mathrm{TiO}_{2}$ surface and strongly indicates a continuous thin liquid film of water. This continuous film is also in excellent agreement with previous Dip and Pull experiments which show a continuous layer of liquid can be achieved and subsequently proven by electrically biasing the liquid layer, showing a complete B.E. shift of the liquid signal, which otherwise would not occur if the liquid film was noncontinuous. It is also noted that no increase of $\mathrm{OH}$ surface groups was noted as a result of the formation of the UTWL water layer on the $\mathrm{TiO}_{2}$ surface.

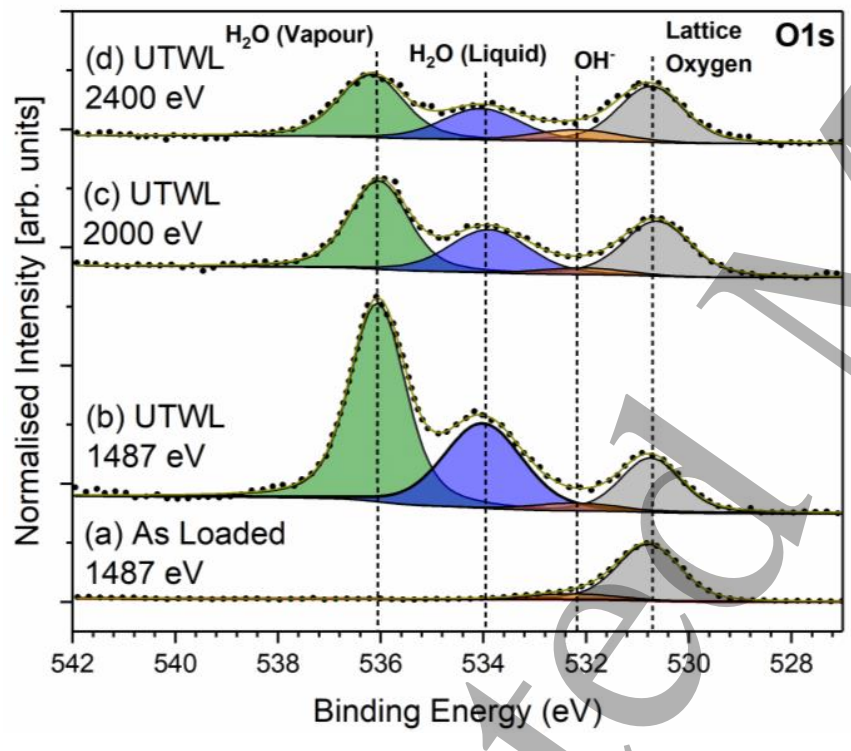

Figure 9: VerSoX NAP-XPS O 1s for (a) As Loaded and photon depth profiling at (b) $1487 \mathrm{eV}$, (c) $2000 \mathrm{eV}$ and (d) $2400 \mathrm{eV}$ of the solid/liquid interface experimental stage. All spectra are normalised to the lattice oxygen peak.

Additionally, Figure 10 (a) displays the Ti $2 \mathrm{p}$ spectra of the sample surface for the "As Loaded" (1487 eV photon energy, at high-vacuum conditions) and final UTWL stage at varying photon energy (Figure $10(\mathrm{~b}-\mathrm{d})$ ). This result is in agreement with the O 1s spectra (Figure 9), showing that the substrate signal is still acquired even through the calculated $12.4 \mathrm{~nm}$ thick water layer. No change is noted in the chemical nature of the Ti $2 \mathrm{p}$ signal of the substrate as a result of the overlayer of liquid water.

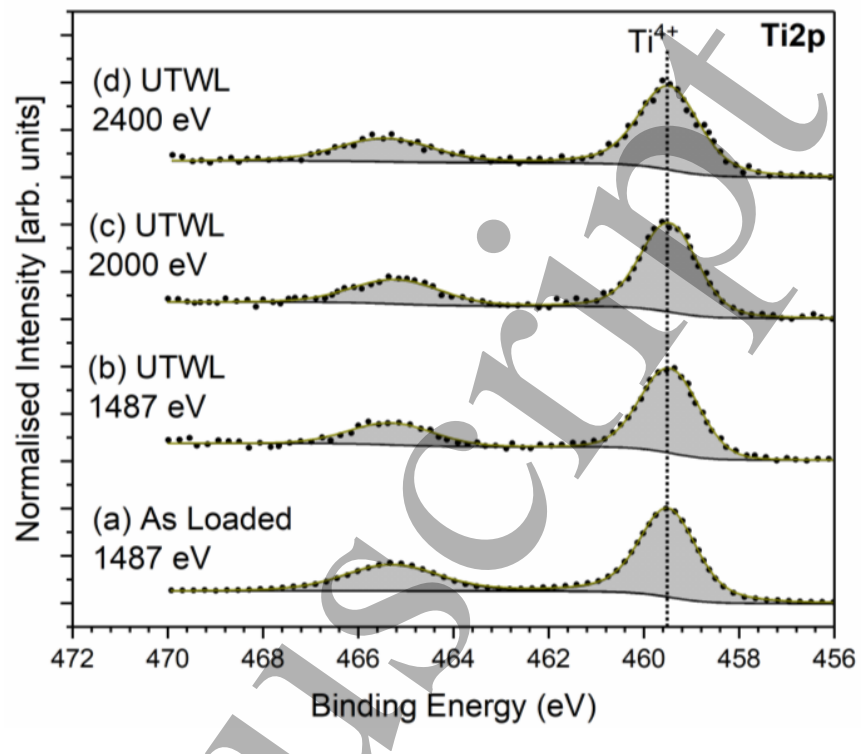

Figure 10: VerSoX NAP-XPS Ti 2p of of $\mathrm{TiO}_{2}(110)$ for (a) As Loaded and photon depth profiling at (b) $1487 \mathrm{eV}$, (c) 2000 $\mathrm{eV}$ and (d) $2400 \mathrm{eV}$ of the solid/liquid interface experimental stage. All spectra are normalised to the Ti $2 p_{3 / 2}$ signal.

(d) UTWL $2400 \mathrm{eV}$

C1s

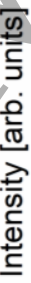

(c) UTWL $2000 \mathrm{eV}$

(b) UTWL $1487 \mathrm{eV}$

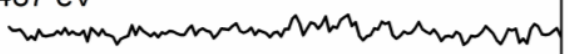

(a) As Loaded $1487 \mathrm{eV}$

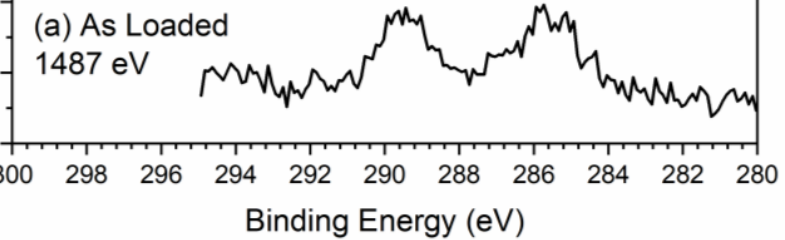

Figure 11: VerSoX NAP-XPS raw intensity C 1s spectra of $\mathrm{TiO}_{2}(110)$ for (a) As Loaded and photon depth profiling at (b) $1487 \mathrm{eV}$, (c) $2000 \mathrm{eV}$ and (d) $2400 \mathrm{eV}$ of the solid/liquid interface experimental stage. All spectra are raw (stacked) signals showing no detectable carbon species at the $\mathrm{TiO}_{2} /$ water interface.

Finally, Figure 11 (a) displays the C1s spectra for "As Loaded" (1487 eV photon energy at high-vacuum conditions) and UTWL stages of varying photon energy 
depth profiling (Figure 11 (b-d)), complementing the Ti 2p and $\mathrm{O}$ 1s spectra. Following UHV sputter-anneal cleaning, it is noted that a very minor carbon signal is present in the surface region of the sample. This minor carbon signal subsequently could not be detected following growth of the UTWL of liquid water over the surface of the $\mathrm{TiO}_{2}$ crystal, even during prolonged scanning at each of the photon energies used. This result indicates that the liquid water layer grown in-situ was of ultra-high purity with negligible carbon contamination introduced during the growth (within the detection limits of XPS).

\section{Conclusions \& Future Outlook}

The Offset Droplet technique has shown to be a versatile method to probe the solid / liquid interface using NAP-XPS. Easy compatibility between both NAP-cell and backfill type NAP-XPS systems has been shown, in addition to the ability to readily implement the set-up at a synchrotron beamline, improving the versatility of the technique.

Photon depth profiling of the as-formed UTWL of liquid water on single crystal $\mathrm{TiO}_{2}$ indicates that the film is likely continuous across the sample analysis region, important for future study of operando reactions at the solid / liquid interface using this method.

Improvements to the technique over past demonstrations in relation to new capillary materials, liquid preparation methods and liquid pump controls have resulted in vastly decreased levels of contamination resulting from exposure of the liquid water to the surface under analysis relative to original proof-of-concept results ${ }^{13}$. Only a minor increase of $\mathrm{C}-\mathrm{C}$ species was noted following advancing and receding of the liquid droplet over the analysis region in the UoM measurements. Further, no increase of any contamination was noted during growth of a relatively thicker UTWL during analysis via synchrotron radiation.

Further to the technical demonstrations and improvements of the Offset Droplet technique, a number of scientific results are raised as a consequence of this work. Firstly, apparent BE shifting of the associated sample and liquid / vapour peaks is noted and appears to be linked to the thickness of water present on the surface of the $\mathrm{TiO}_{2}$ surface (previously linked to band-bending at the surface of the $\mathrm{TiO}_{2}$ ). Although no $\mathrm{BE}$ shifted values are reported in this study (as it is deemed outside the scope of the study), future work can utilise the staggered thickness increase of liquid water growth, demonstrated at the VerSoX beamline, to track the associated shift relative to the growth of the thickness of the water layer in order to give highly informed detail of the solid / liquid interface for the $\mathrm{TiO}_{2} /$ water interface.

Secondly, two growth mechanisms of forming an UTWL over a surface have been proposed. The initial method creates a UTWL by advancing the droplet over the analysis region and subsequently receding it, similar to the Dip and
Pull method. The second mechanism is described as constraining the liquid droplet adjacent to the analysis area and allowing the UTWL to form over time either via a condensation mechanism (akin to an in-situ "Cold Finger" experiment ${ }^{7}$ ) or spreading of the tail end of the droplet into the analysis region. Both mechanisms give insight into the ability to form UTWL of liquids in vacuum environments however further work must be performed in order to understand the true mechanism behind formation of these UTWLs. The Dip and Pull method assumes that the UTWL formed is the tail of the liquid meniscus formed from dipping and subsequently pulling a long sample into/from a beaker of liquid in-situ ${ }^{12}$. However, we believe that real picture may be more complex, with local condensation from water vapour also playing a role.

Another apparent point of difference is that in results obtained both here and in previous literature ${ }^{13}$. The Offset Droplet method has shown either a comparable thickness of liquid or a smaller thickness value despite the bulk of the liquid being closer to the analysis area relative to the Dip and Pull method ( $\angle 00$ microns for the offset droplet instead of $\sim 2 \mathrm{~cm}$ for Dip and Pull) ${ }^{24}$. This variation in meniscus tail thickness from bulk liquid to analysis area could be explained as a result of the specific hydrophobicity of the sample surface however it seems unlikely given the high level of sample surface cleanliness achieved during execution of both methods. Future work in relation to the mechanisms of UTWL formation and control could lead to improvements of both the Dip and Pull and Offset Droplet techniques which complement each other in terms of abilities and associated experimental strengths.

Future work is planned to utilise the Offset Droplet Technique in order to create UTWL of pure liquid water on $\mathrm{TiO}_{2}$ surfaces in order to study photo-degradation of adsorbed hydrocarbon species at the solid / liquid interface by controlled introduction of hydrocarbon species in the presence of UV radiation. In addition, operando electrochemical experiments at the solid / liquid interface have been advanced by utilising a modified Offset Droplet set-up (with counter and reference electrodes) in order to probe electrochemical reactions at the electrified solid / liquid interface, similar to previous reports involving the Dip and Pull method ${ }^{15}$.

\section{Acknowledgements}

The authors wish to acknowledge the Henry Royce Institute for continued support in relation to equipment access and support in system modifications to the SPECS DeviSim NAP-XPS system located at the University of Manchester (funded through EPSRC grants EP/R00661X/1 and EP/P025021/1). ASW would like to thank the EPSRC for funding (EP/S004335/1). Diamond Light Source facilities were provided under beamtime S123855. SPECS Surface 
Nano Analysis $\mathrm{GmbH}$ are acknowledged for their continued support in relation to integration of the Offset droplet technique within the University of Manchester SPECS NAPXPS system. The staff of beamline B07 are acknowledged for assistance with integration and experiments using the Offset Droplet cell. C.Byrne wishes to acknowledge the support of Prof S. Andbar and Dr. Salisbury groups for supplies of dilute ethanol solutions, critical to the ongoing insitu UTWL work.

\section{References}

${ }^{1}$ A. Fujishima, X. Zhang, and D.A. Tryk, Surface Science Reports 63, 515 (2008).

2 J. Schneider, M. Matsuoka, M. Takeuchi, J. Zhang, Y. Horiuchi, M. Anpo, and D.W. Bahnemann, Chem. Rev. 114, 9919 (2014).

${ }^{3}$ M. Grätzel, Journal of Photochemistry and Photobiology C: Photochemistry Reviews 4, 145 (2003).

${ }^{4}$ R. Lindsay, S. Tomić, A. Wander, M. García-Méndez, and G. Thornton, J. Phys. Chem. C 112, 14154 (2008).

${ }^{5}$ C. Lun Pang, R. Lindsay, and G. Thornton, Chem. Soc. Rev. 37, 2328 (2008).

${ }^{6}$ D.C. Grinter, T. Woolcot, C.-L. Pang, and G. Thornton, J. Phys. Chem. Lett. 5, 4265 (2014).

${ }^{7}$ J. Balajka, M.A. Hines, W.J.I. DeBenedetti, M. Komora, J. Pavelec, M. Schmid, and U. Diebold, Science 361, 786 (2018).

${ }^{8}$ M.C. Biesinger, L.W.M. Lau, A.R. Gerson, and R.St.C. Smart, Applied Surface Science 257, 887 (2010).

${ }^{9}$ W. Göpel, J.A. Anderson, D. Frankel, M. Jaehnig, K. Phillips, J.A. Schäfer, and G. Rocker, Surface Science 139, 333 (1984).

${ }^{10}$ U. Gelius and K. Siegbahn, Faraday Discuss. Chem. Soc. 54, 257 (1972).

${ }^{11}$ H. Siegbahn, S. Svensson, and M. Lundholm, Journal of Electron Spectroscopy and Related Phenomena 24, 205 (1981).

${ }^{12}$ S. Axnanda, E.J. Crumlin, B. Mao, S. Rani, R. Chang, P.G. Karlsson, M.O.M. Edwards, M. Lundqvist, R. Moberg, P. Ross, Z. Hussain, and Z. Liu, Sci Rep 5, 9788 (2015).

${ }^{13}$ S.G. Booth, A.M. Tripathi, I. Strashnov, R.A.W. Dryfe, and A.S. Walton, J. Phys.: Condens. Matter 29, 454001 (2017).

${ }^{14}$ M. Favaro, B. Jeong, P.N. Ross, J. Yano, Z. Hussain, Z. Liu, and

E.J. Crumlin, Nat Commun 7, 12695 (2016).

${ }^{15}$ K.A. Stoerzinger, M. Favaro, P.N. Ross, Z. Hussain, Z. Liu, J.

Yano, and E.J. Crumlin, Top Catal 61, 2152 (2018).

${ }^{16}$ Z. Novotny, D. Aegerter, N. Comini, B. Tobler, L. Artiglia, U.

Maier, T. Moehl, E. Fabbri, T. Huthwelker, T.J. Schmidt, M.

Ammann, J.A. van Bokhoven, J. Raabe, and J. Osterwalder, Review of Scientific Instruments 91, 023103 (2020).

${ }^{17}$ G. Held, F. Venturini, D.C. Grinter,P. Ferrer, R. Arrigo, L.

Deacon, W. Quevedo Garzon, K. Roy, A. Large, C. Stephens, A.

Watts, P. Larkin, M. Hand, H. Wang, L. Pratt, J.J. Mudd, T.

Richardson, S. Patel, M. Hillman, and S. Scott, J Synchrotron Rad 27, 1153 (2020).

${ }^{18}$ L.E. Walle, A. Borg, P. Uvdal, and A. Sandell, Phys. Rev. B 80, 235436 (2009)

${ }^{19}$ L.-Q. Wang, D.R. Baer, M.H. Engelhard, and A.N. Shultz, Surface Science 344, 237 (1995).

${ }^{20}$ M. Favaro, F. Abdi, E. Crumlin, Z. Liu, R. van de Krol, and D. Starr, Surfaces 2, 78 (2019).

${ }^{21}$ M.J. Makowski, R.P. Galhenage, J. Langford, and J.C.

Hemminger, J. Phys. Chem. Lett. 7, 1732 (2016).
22 J.F. Moulder, W.F. Stickle, P.E. Sobol, and K.D. Bomben, Handbook of X-Ray Photoelectron Spectroscopy (Perkin-Elmer Corp, Eden Prairie, Mn, 1992).

${ }^{23}$ G. Ketteler, S. Yamamoto, H. Bluhm, K. Andersson, D.E. Starr, D.F. Ogletree, H. Ogasawara, A. Nilsson, and M. Salmeron, J. Phys. Chem. C 111, 8278 (2007).

${ }^{24}$ O. Karslıŏlu, S. Nemšák, I. Zegkinoglou, A. Shavorskiy, M. Hartl, F. Salmassi, E.M. Gullikson, M.L. Ng, C. Rameshan, B. Rude, D. Bianculli, A.A. Cordones, S. Axnanda, E.J. Crumlin, P.N. Ross, C.M. Schneider, Z. Hussain, Z. Liu, C.S. Fadley, and H. Bluhm, Faraday Discuss. 180, 35 (2015). 